\title{
Swapping path-spin intraparticle entanglement onto spin-spin interparticle entanglement
}

\author{
S. Adhikar* and A. S. Majumdari \\ S. N. Bose National Centre for Basic Sciences, Salt Lake, Kolkata 700 098, India \\ Dipankar Homf and A. K. Pan \\ CAPSS, Dept. of Physics, Bose Institute, Salt Lake, Kolkata-700091, India
}

(Dated: October 24, 2018)

\begin{abstract}
Based on a scheme that produces an entanglement between the spin and the path variables of a single spin-1/2 particle (qubit) using a beam-splitter and a spin-flipper, we formulate a procedure for transferring this intraparticle hybrid entanglement to an interparticle entanglement between the spin variables of two other spatially separated spin- $1 / 2$ particles which never interact with each other during the entire process. This procedure of entanglement swapping is accomplished by a Mach-Zehnder setup in conjunction with the Stern-Gerlach measuring device and by using suitable unitary operations. The proposed protocol, thus, enables the use of intraparticle entanglement as a resource - a feature that has remained unexplored.
\end{abstract}

PACS numbers: 03.65.Ud

Over the years, quantum entanglement has been increasingly recognised as a key ingredient in the information theoretic processes involving storage and distribution of information among the fundamental constituents of the world [1]. The first profound implication of entanglement for quantum foundations was noticed way back in 1935 using position and momentum variables [2], and was later extended to the discrete spin variables [3]. In recent times there has been significant development of the theory of entanglement for systems described by various types of Hilbert spaces, such as those corresponding to discrete variables 4 as well as for those pertaining to continuous variables [5]. Several powerful information processing protocols such as cryptography [], densecoding [7], quantum teleportation [8], and entanglement swapping [9] have been developed for the spin entangled states, as well as for the position-momentum entangled states [10, 11].

The study of various aspects of entanglement and the associated applications in the context of information theory promises to provide insights into a wide range of diverse phenomena such as phase transitions in condensed matter systems [12] and black hole physics [13]. Against this backdrop, the investigation of hybrid entanglement between the physical variables in mutually different Hilbert spaces such as those corresponding to the path (or linear momentum) variables on the one hand, and spin variables on the other, is of special relevance. Although the theoretical framework of quantum mechanics allows for the existence of hybrid entangled states involving Hilbert spaces with distinct properties, the possibility of physical realization of such states has not been much explored and is only beginning to be appreciated [14].

Another interesting recent line of development is based on the idea of generation of intraparticle entanglement between the different degrees of freedom of the same particle. The entanglement between polarization and linear momentum of a single photon [15], and also that between polarization and angular momentum of a single photon 16] have been demonstrated experimentally. The idea of creating an entanglement between the path and the spin degrees of freedom for a single spin-1/2 particle was first proposed in order to demonstrate a testable incompatibility between quantum mechanics and noncontextual realist models 17]. Subsequently, such a path-spin hybrid entangled state for a single neutron has been realized experimentally [18]. Recently, an interesting application of hybrid intraparticle entanglement has been discussed in the context of neutrino oscillations [19]. There have also been studies that use the notion of the intraparticle entanglement for demonstrating nonlocality of a single photon [20].

Now, an important point to note is that since intraparticle entanglement between the different degrees of freedom is confined to a single particle, such an entanglement should be relatively easier to preserve, at least in principle, against decoherence effects. It is then natural to ask the question whether this type of hybrid entanglement between the different degrees of freedom of the same particle can be used as a resource for information processing.

At the outset, the above idea may seem difficult to implement, since the entanglement considered is not shared nonlocally between two spatially separated regions in a way that is amenable to be exploited as a resource. One way out, however, would be to transfer a given intraparticle entanglement onto an entanglement between the appropriate degrees of freedom of two spatially separated particles.

It is from this perspective that in this paper we demonstrate how the path-spin entanglement of a single spin- 
$1 / 2$ particle can be transferred to the spin-spin entanglement involving two spin- $1 / 2$ particles which remain spatially separated and non-interacting with each other during the entire procedure by which this transfer of entanglement is achieved. In order to outline the realizability of such a scheme, we first discuss a method to generate an intraparticle hybrid path-spin entanglement using a beam-splitter and a spin-flipper. Then, our protocol for entanglement swapping becomes implementable with the help of two additional spin- $1 / 2$ particles that never interact with each other during the entire process. For this, two separate parties (Alice and Bob) perform a series of operations that include unitary transformations together with appropriate measurements involving the Stern-Gerlach devices, followed by the use of classical communication that enables to eventually transfer the information content of an intraparticle path-spin entangled state to the spin-spin entanglement pertaining to two spatially separated spin- $1 / 2$ particles. 1.

The proposed scheme is pictorially illustrated in Figure

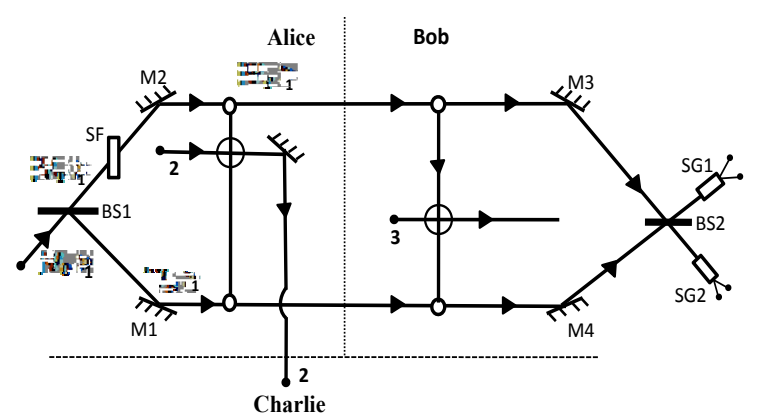

FIG. 1: A spin-1/2 particle (labelled as particle 1) falls on the beam-splitter BS1. A spin-flipper is placed along the transmitted channel. A CNOT operation is performed by Alice involving particle ' 2 ' and the particle ' 1 ' which is subsequently transported to Bob. While the particle ' 2 ' is sent to Charlie, Bob recombines the reflected and the transmitted channels corresponding to particle 1 by using the beam-splitter BS2, and then Bob performs spin measurements using the Stern-Gerlach devices SG1 and SG2. According to the measurement results, Bob performs appropriate unitary transformations on the states of the two qubits ' 1 ' and ' 3 ' that are with him. As a result, the two spatially separated qubits ' 2 ' and ' 3 ' with Charlie and Bob respectively get entangled although they have never interacted with each other.

Let us consider a spin- $1 / 2$ particle (say, a neutron, labelled as particle 1) that has an initial spin polarized state along the $+\widehat{z}$ - axis (denoted by $\left|\uparrow_{z}\right\rangle$ ). Taking into consideration its path (or position) variables, the joint path-spin state can be written as

$$
\left.\left|\Psi_{0}>_{p s}=\right| \psi_{0}\right\rangle_{p} \otimes\left|\uparrow_{z}\right\rangle_{s}
$$

where the subscripts $p$ and $s$ refer to the path and the spin variables respectively. The ensemble of neutrons corresponding to $\mid \Psi_{o}>_{p s}$ are with Alice, incident on a beam-splitter (BS1) whose transmission and reflection probabilities are $|\alpha|^{2}$ and $|\beta|^{2}$ respectively. Any given incident particle can then emerge along either the reflected or the transmitted channel corresponding to the state designated by $\left|\psi_{R}\right\rangle$ or $\left|\psi_{T}\right\rangle$ respectively.

Here we recall that for any given lossless beam-splitter, arguments using the unitarity condition show that for the particles incident on the beam-splitter, the phase shift between the transmitted and the reflected states of particles is essentially $\pi / 2[21]$. For simplifying our treatment, we take the transmission and reflection amplitudes of BS1 and BS2 to be real quantities where $\alpha^{2}+\beta^{2}=1$. Note that the beam-splitter acts only on the path-states without affecting the spin-state of the particles, while in our argument a crucial role is played by the mutually orthogonal path states $\left|\psi_{R}\right\rangle$ and $\left|\psi_{T}\right\rangle$ which are eigenstates of the projection operators $P\left(\psi_{R}\right)$ and $P\left(\psi_{T}\right)$ respectively.

These projection operators can be regarded as corresponding to the observables that pertain to the determination of 'which channel' a particle is found to be in. For example, the results of such a measurement for the reflected (transmitted) channel with binary alternatives are given by the eigenvalues of $P\left(\psi_{R}\right)\left(P\left(\psi_{T}\right)\right)$; the eigenvalue $+1(0)$ corresponds to a neutron being found (not found) in the channel represented by $\left|\psi_{R}\right\rangle\left(\left|\psi_{T}\right\rangle\right)$.

The state of a particle emergent from BS1 can then be written as

$$
\left|\Psi_{0}>_{p s} \rightarrow\right| \Psi_{1}>_{p s}=\left(\alpha\left|\psi_{T}\right\rangle_{p}+i \beta\left|\psi_{R}\right\rangle_{p}\right) \otimes\left|\uparrow_{z}\right\rangle_{s}
$$

The state vectors $\left|\psi_{T}\right\rangle_{p},\left|\psi_{R}\right\rangle_{p},\left|\uparrow_{z}\right\rangle_{s}$, and $\left|\downarrow_{z}\right\rangle_{s}$ can be represented as

$$
\begin{array}{r}
\left|\psi_{T}\right\rangle_{p} \equiv\left(\begin{array}{l}
0 \\
1
\end{array}\right),\left|\psi_{R}\right\rangle_{p} \equiv\left(\begin{array}{l}
1 \\
0
\end{array}\right), \\
\left|\uparrow_{z}\right\rangle_{s} \equiv|0\rangle_{s}=\left(\begin{array}{l}
0 \\
1
\end{array}\right),\left|\downarrow_{z}\right\rangle_{s} \equiv|1\rangle_{s}=\left(\begin{array}{l}
1 \\
0
\end{array}\right)
\end{array}
$$

At this stage, we may stress that although here a single particle is considered at a time, the dichotomic path and spin variables enable it to be viewed effectively as two qubits. Using the above notation, the state (2) can be written as

$$
\mid \Psi_{1}>_{p s}=\left(\begin{array}{c}
0 \\
i \beta \\
0 \\
\alpha
\end{array}\right)
$$

Next, let us suppose that the particles in the channel corresponding to $\left|\psi_{T}\right\rangle$ pass through a spin-flipper (SF) (that contains a uniform magnetic field along, say, the $+\widehat{x}$-axis) which flips the state $|\uparrow\rangle_{z}$ to $|\downarrow\rangle_{z}$. As a consequence of this insertion of a spin-flipper in one of the channels, the particle 1 with Alice has now the path-spin 
entangled state given by

$$
\begin{aligned}
\mid \Psi>_{p s} & =\alpha\left|\uparrow_{z}\right\rangle_{s} \otimes\left|\psi_{T}\right\rangle_{p}+i \beta\left|\downarrow_{z}\right\rangle_{s} \otimes\left|\psi_{R}\right\rangle_{p} \\
& \equiv \alpha|0\rangle_{s} \otimes\left|\psi_{T}\right\rangle_{p}+i \beta|1\rangle_{s} \otimes\left|\psi_{R}\right\rangle_{p}
\end{aligned}
$$

Note that the above path-spin entanglement, given by Eq. 15, is between the spin variables and the pseudospin like path observables of a spin- $1 / 2$ particle - this is what we call the hybrid 'intraparticle entanglement' that is distinct from the usually discussed 'interparticle entanglement', say, between the spin variables of two spatially separated particles, as well as is different from the recently discussed form of 'hybrid entanglement' 14] between the polarization of one photon and the spin of another spatially separated photon.

Next, we assume that Alice possesses another particle (labelled as particle 2) which is in the up-spin state $\mid \uparrow$ \rangle$_{2} \equiv|0\rangle_{2}$. The total state is now the combination of the path-spin entangled state and the spin state $|0\rangle_{2}$, which can be written as

$$
\begin{aligned}
|\Omega\rangle_{p s 2} & =|\Psi\rangle_{p s} \otimes|0\rangle_{2} \\
& =\alpha|00\rangle_{s 2} \otimes\left|\psi_{T}\right\rangle_{p}+i \beta|10\rangle_{s 2} \otimes\left|\psi_{R}\right\rangle_{p}
\end{aligned}
$$

At this stage, we consider that Alice performs a suitable two qubit CNOT operation on the qubits 's' and ' 2 ' by taking the qubit ' $\mathrm{s}$ ' as a source and the qubit ' 2 ' as a target qubit. Then, as a result, the transformed state is given by

$$
|\Phi\rangle_{p s 2}=\alpha|00\rangle_{s 2} \otimes\left|\psi_{T}\right\rangle_{p}+i \beta|11\rangle_{s 2} \otimes\left|\psi_{R}\right\rangle_{p}
$$

Next, let us suppose that Alice sends the particle ' 1 ' (embodying the qubits 'p' and 's') to her distant partner Bob who possesses another particle ' 3 ' with the spin polarised state $\left|\uparrow_{z}\right\rangle$. After receiving the particle '1', Bob performs a CNOT operation on the qubits 's' and '3' on his side by taking the qubit 's' as a source and the qubit '3' as a target qubit. Taking into account Bob's operation, the four-qubit joint (Alice-Bob) state can be written as

$$
|\Psi\rangle_{p s 23}=\alpha|000\rangle_{s 23} \otimes\left|\psi_{T}\right\rangle_{p}+i \beta|111\rangle_{s 23} \otimes\left|\psi_{R}\right\rangle_{p}
$$

where note that the qubits 'p', 's' and ' 3 ' are physically with Bob whereas the qubit ' 2 ' with Alice is sent to another distant party Charlie.

Subsequently, the particle 1 passing through either of the two channels $\left|\psi_{T}\right\rangle\left(\left|\psi_{R}\right\rangle\right)$ is reflected by the mirrors M2 and M3 ( M1 and M4) - these reflections do not lead to any net relative phase shift between the channels $\left|\psi_{R}\right\rangle$ and $\left|\psi_{T}\right\rangle$. Bob then uses a $50-50$ beam splitter (BS2) to recombine the two paths. The states $\left|\psi_{T}\right\rangle_{p}$ and $\left|\psi_{R}\right\rangle_{p}$ are transformed by BS2 to

$$
\begin{aligned}
\left|\psi_{T}\right\rangle_{p} & \rightarrow \frac{1}{\sqrt{2}}\left(i\left|\psi_{T}^{\prime}\right\rangle_{p}+\left|\psi_{R}^{\prime}\right\rangle_{p}\right) \\
\left|\psi_{R}\right\rangle_{p} & \rightarrow \frac{1}{\sqrt{2}}\left(\left|\psi_{T}^{\prime}\right\rangle_{p}+i\left|\psi_{R}^{\prime}\right\rangle_{p}\right)
\end{aligned}
$$

In writing Eq. (9) we have taken into account a relative phase shift of $\pi / 2$ between the states $\left|\psi_{R}^{\prime}\right\rangle$ and $\left|\psi_{T}^{\prime}\right\rangle$ that arises because of the reflection from BS2. Using the transformation (9), the four-qubit state represented by Eq.(8) evolves to

$$
\begin{aligned}
|\Psi\rangle_{p s 23} & =\frac{i}{\sqrt{2}}\left[\left(\alpha|000\rangle_{s 23}+\beta|111\rangle_{s 23}\right) \otimes\left|\psi_{T}^{\prime}\right\rangle_{p}\right] \\
& +\frac{1}{\sqrt{2}}\left[\left(\alpha|000\rangle_{s 23}-\beta|111\rangle_{s 23}\right) \otimes\left|\psi_{R}^{\prime}\right\rangle_{p}\right](10)
\end{aligned}
$$

Beyond the beam-splitter BS2, the Stern-Gerlach apparatuses denoted by SG1 and SG2 are placed by which the qubit ' $\mathrm{s}$ ' undergoes a unitary transformation $|0\rangle_{s} \rightarrow \frac{1}{\sqrt{2}}\left(|0\rangle_{s}+|1\rangle_{s}\right),|1\rangle_{s} \rightarrow \frac{1}{\sqrt{2}}\left(|0\rangle_{s}-|1\rangle_{s}\right)$. Now, depending upon which of the two paths $\left|\psi_{T}^{\prime}\right\rangle_{p}$ or $\left|\psi_{R}^{\prime}\right\rangle_{p}$ are taken by Bob's particle 1, there exist the following possibilities:

Case-I: If Bob's particle 1 travels along $\left|\psi_{T}^{\prime}\right\rangle_{p}$ then after its interaction with the Stern-Gerlach apparatus, the reduced three-qubit state is given by

$$
\begin{array}{r}
|\Psi\rangle_{s 23}=\frac{1}{\sqrt{2}}\left[|0\rangle_{s} \otimes\left(\alpha|00\rangle_{23}+\beta|11\rangle_{23}\right)\right. \\
+|1\rangle_{s} \otimes\left(\alpha|00\rangle_{23}-\beta|11\rangle_{23}\right]
\end{array}
$$

In this case, the following are the possibilities:

(i) If Bob's measurement outcome is $|0\rangle_{s}$, then the twoqubit state for the paticles 2 and 3 is given by

$$
\left|\chi_{1}\right\rangle_{23}=\alpha|00\rangle_{23}+\beta|11\rangle_{23}
$$

(ii) If the measurement outcome is $|1\rangle_{s}$, then the twoqubit state at for 2 and 3 reduces to

$$
\left|\chi_{2}\right\rangle_{23}=\alpha|00\rangle_{23}-\beta|11\rangle_{23}
$$

Case-II: If Bob's particle 1 travels along $\left|\psi_{R}^{\prime}\right\rangle_{p}$, then after its interaction with the Stern-Gerlach apparatus, the reduced three-qubit state is given by

$$
\begin{array}{r}
|\Psi\rangle_{s 23}=\frac{1}{\sqrt{2}}\left[|0\rangle_{s} \otimes\left(\alpha|00\rangle_{23}-\beta|11\rangle_{23}\right)\right. \\
+|1\rangle_{s} \otimes\left(\alpha|00\rangle_{23}+\beta|11\rangle_{23}\right]
\end{array}
$$

In this case, the following are the possibilities:

(i) If Bob's measurement outcome is $|0\rangle_{s}$, then the twoqubit state for the particle 2 and 3 is given by

$$
\left|\chi_{3}\right\rangle_{23}=\alpha|00\rangle_{23}-\beta|11\rangle_{23}
$$

(ii) If the measurement outcome is $|1\rangle_{s}$, then the twoqubit state for 2 and 3 reduces to

$$
\left|\chi_{4}\right\rangle_{23}=\alpha|00\rangle_{23}+\beta|11\rangle_{23}
$$


At this final stage, all that remains for Bob to do is to perform suitable unitary transformations to create an entangled state of the qubits ' 2 ' and ' 3 ' that are with Bob and Charlie respectively - this state being a replica (in terms of the information content) of the path-spin entangled state (5) originally possessed by Alice. The required unitary operations corresponding to each measurement outcome are given in the following Table:

Table-1:

\begin{tabular}{|c|c|c|}
\hline Path state & $\begin{array}{c}\text { Measurement } \\
\text { outcome }\end{array}$ & $\begin{array}{c}\text { Unitary transformation } \\
\text { performed on qubits 2 and 3 }\end{array}$ \\
\hline$\left|\psi_{T}^{\prime}\right\rangle_{p}$ & $|0\rangle_{s}$ & $I \otimes S$ \\
& $|1\rangle_{s}$ & $I \otimes \sigma_{z} \cdot S$ \\
\hline$\left|\psi_{R}^{\prime}\right\rangle_{p}$ & $|0\rangle_{s}$ & $I \otimes \sigma_{z} \cdot S$ \\
& $|1\rangle_{s}$ & $I \otimes S$ \\
\hline
\end{tabular}

Here $\sigma_{z}$ is the Pauli spin matrix and $S$ denotes the phase gate defined as

$$
S=\left(\begin{array}{ll}
1 & 0 \\
0 & i
\end{array}\right)
$$

Thus, after performing the unitary operations as indicated in Table 1, for each measurement outcome, Bob and Charlie are ultimately left with sharing a two-qubit spinspin interparticle entangled state which has the same information content as that embodied in the original pathspin intraparticle entangled state used by Alice.

Therefore, the upshot of our above demonstration is that the "swapping" of the intraparticle entanglement encoded in particle ' 1 ' into useful interparticle entanglement of the spatially separated particles ' 2 ' and ' 3 ' is achieved by satisfying the following key condition: Particles ' 2 ' and ' 3 ' are kept separated and they never interact with each other during the entire process. In other words, using our scheme, the particles ' 2 ' and ' 3 ' can get entangled by only letting the particle ' 1 ' to interact independently with the particles ' 2 ' and ' 3 ', without the need of having ' 2 ' and ' 3 ' in the same place and without the need for a joint unitary operation on ' 2 ' and ' 3 '.

Before concluding, we may note that Cubitt et al. [22] had suggested a scheme using which two distant particles can be entangled by continuous interaction with a mediating(ancilla) particle that never itself becomes entangled. It should, however, be evident that our scheme is formulated in a way that is basically different from that proposed by Cubitt et al. because, in essence, what our work reveals is the procedure by which the information encoded in the entanglement between two different degrees of freedom of the same particle can be transferred across a distance by creating an entangled spin state of two spatially separated particles. This procedure, therefore, opens up the possibility that the hybrid entanglement at the level of a single particle can be used to perform interesting information processing tasks. Such a possibility needs to be explored by further detailed studies.

It is worth stressing the ubiquity of the path (or linear momentum) degrees of freedom in any experimental setup using particles/photons. In our proposed scheme, it is the pseudo-spin like path variable that has been invoked to first generate the path-spin entanglement for a single qubit. Then, using a suitable setup, this entangled state is transferred to an entanglement between the spins of two qubits which remain spatially separated and never interact with each other. While two CNOT operations have been used in two different stages during the process, they are essentially local operations that ultimately enable nonlocal sharing of entanglement between two spatially separated particles without requiring them to be subjected to any global unitary operation. It may be noted here that it has been proposed in 23] that the path information could be used as a qubit source to simulate a duality quantum computer, thus providing a possible application of our scheme for such purposes. Further, note that, although the suggested protocol is demonstrated for spin-1/2 particles, it may also be implemented using, say, photons and suitable optical devices. Finally, this demonstration underscores the usability of path-spin entanglement pertaining to a single particle [17, 18] as a physical resource, thereby exemplifying the power of hybrid entanglement [14] as a fundamental concept, independent of any particular physical realization of Hilbert space.

Acknowledgements: ASM and DH acknowledge support from the DST Project SR/S2/PU-16/2007. DH also thanks the Centre for Science, Kolkata for support.

* satyabrata@bose.res.in

$\dagger$ archan@bose.res.in

¥ dhome@bosemain.boseinst.ac.in

$\S$ apan@bosemain.boseinst.ac.in

[1] A. Peres, "Quantum Theory: Concepts and Methods", (Kluwer Academic Publishers, The Netherlands, 1995); D. Home, "Conceptual Foundations of Quantum Physics: An overview from Modern Perspectives", (Plenum, New York, 1997);H. K. Lo, S. Popescu and T. Spiller, eds "Introduction to Quantum Computation and Information" (World Scientific, Singapore, 1998); M. Nielsen and I. Chuang, "Quantum Computation and Quantum Information",(Cambridge University Press, Cambridge (2000); D. Bouwmeester, A. Ekert, and A. Zeilinger, eds. "The Physics of Quantum Information: Quantum Cryptography, Quantum Teleportation, Quantum Computation" (Springer-Verlag, Berlin, 2001).

[2] A. Einstein, B. Podolsky and N. Rosen, Phys. Rev. 47, 777 (1935).

[3] D. Bohm, "Quantum Theory", (Prentice-Hall, Englewood Cliffs, NJ, 1951).

[4] R. Horodecki, P. Horodecki, M. Horodecki and K. 
Horodecki, arXiv: quant-ph/0702225

[5] S. L. Braunstein and P. van Loock, Rev. Mod. Phys. 77, 513 (2005); J. Laurat, G. Keller, J-A Oliveira-Huguenin, C. Fabre, T. Coudreau, A. Serafini, G. Adesso and F. Illuminati, Journal of Optics B 7, S577 (2005).

[6] C. H. Bennett and G. Brassard, Proceedings of IEEE International Conference on Computers,Systems and Signal processing, pages 175-179, Bangalore, India, 1984; A. K. Ekert, Phys. Rev. Lett. 67, 661 (1991).

[7] C. H. Bennett and S. J. Wiesner, Phys. Rev. Lett. 69, 2881 (1992).

[8] C. H. Bennett, G. Brassard, C. Crepeau, R. Jozsa, A. Peres, and W. K. Wootters, Phys. Rev. Lett. 70, 1895 (1993).

[9] M. Zukowski, A. Zeilinger, M. A. Horne and A. K. Ekert, Phys. Rev. Lett. 71, 4287 (1993); S. Bose, V. Vedral and P. L. Knight, Phys. Rev. A 57, 822 (1998).

[10] L. Vaidman, Phys. Rev. A 49, 1473 (1994); S. L. Braunstein and H. J. Kimble, Phys. Rev. A 49, 1567 (1994); S. Adhikari, A. S. Majumdar and N. Nayak, Phys. Rev. A 77, 012337 (2008); ibid. 77, 042301 (2008).

[11] S. L. Braunstein and P. V. Loock, Rev. Mod. Phys. 77, 513 (2005).

[12] D. Aharonov, Phys. Rev. A 62 , 062311 (2000); L. -A. Wu, M. S. Sarandy, D. A. Lidar and L. J. Sham, Phys. Rev. A 74, 052335 (2006).
[13] S. L. Braunstein and A. K. Pati, Phys. Rev. Lett. 98, 080502 (2007); M. Arzano, A. Hamma and S. Severini, arXiv:0806.2145

[14] M. Zukowski and A. Zeilinger, Phys. Lett. A 155, 69 (1991); X.-S. Ma, A. Qarry, J. Kofler, T. Jennewein and A. Zeilinger, Phys. Rev. A 79, 042101 (2009).

[15] M. Michler, H. Weinfurter, and M. Zukowski, Phys. Rev. Lett. 84, 5457 (2000).

[16] J. T. Barreiro, T.-C. Wei and P. G. Kwiat, Nat. Phys. 4, 282 (2008).

[17] S. Basu, S. Bandyopadhyay, G. Kar and D. Home, Phys. Lett A 279, 281(2001).

[18] Y. Hasegawa, R. Loidl, G. Badurek, M. Baron and H. Rauch, Nature 425, 45 (2003).

[19] M. Blasone, F. DellAnno, S. De Siena and F. Illuminati, Europhys. Lett. 85, 50002(2009).

[20] S. J. van Enk, Phys. Rev. A, 72 064306(2005); J. Dunningham and V. Vedral, Phys. Rev. Lett., 99 180404(2007).

[21] A. Zeilinger, Am. J. Phys. 49, 882 (1981).

[22] T. S. Cubitt, F. Verstraete, W. Duer, and J. I. Cirac, Phys. Rev. Lett. 91, 037902(2003).

[23] Gui Lu Long, Commun. Theor. Phys. 45, 825 (2006); Gui Lu Long and Yang Liu, Commun. Theor. Phys. 50, 1302(2008). 